\title{
Transplant-Ineligible Symptomatic but Indolent Multiple Myeloma Shows Better Prognosis with Conventional Agents
}

\author{
Aya Nakaya Shinya Fujita Atsushi Satake Takahisa Nakanishi \\ Yoshiko Azuma Yukie Tsubokura Akiko Konishi Masaaki Hotta \\ Hideaki Yoshimura Kazuyoshi Ishii Tomoki Ito Shosaku Nomura
}

First Department of Internal Medicine, Kansai Medical University, Hirakata, Japan

\section{Keywords}

Indolent - Symptomatic myeloma - Conventional therapy - Absence of deep response

\section{Abstract}

The survival of multiple myeloma patients has improved significantly over the last several decades. However, the median overall survival of these patients remains less than 5 years. In this report, we discuss 4 cases of multiple myeloma patients that showed long survival. Interestingly, these patients had severe organ damage at diagnosis, used only conventional agents, and did not always show deep response. Although current guidelines recommend novel agents to achieve deep response, the current cases suggest that some multiple myeloma patients may not need intensive treatment. Here, we discuss 4 cases of symptomatic but indolent transplant-ineligible myeloma. 


\section{Case Reports in Oncology}

\section{Case Reports}

Case 1

A 68-year-old Japanese woman with cerebral infarction was diagnosed with multiple myeloma IgGк, Durie-Salmon stage IIIA, ISS II. At diagnosis, she had a performance status (PS) of 2; bone marrow plasmacytosis was $34.6 \%$ and serum IgG was 7,169 mg/dL. She had anemia $(\mathrm{Hb} 8.0 \mathrm{~g} / \mathrm{dL})$ and osteolysis bone lesion. No cytogenetic abnormalities were detected by fluorescent in situ hybridization (FISH). She was started with melphalan and prednisolone (MP), and plateau phase was achieved after 6 courses of MP. After plateau, MP was given intermittently until progression of disease. During the treatment period, she had no severe adverse events and maintained a stable disease (SD) without deep response. Even after the patient was approved to receive novel agents, she refused intensive treatment because of cerebral infarction sequelae. She survived for 6.7 years (Table 1).

Case 2

A 78-year-old Japanese man was diagnosed with multiple myeloma IgGк, Durie-Salmon stage IIIB, ISS II. At diagnosis, he had a PS of 1; bone marrow plasmacytosis was $12.7 \%$ and serum IgG was 3,164 mg/dL. He had mild anemia (Hb $11.8 \mathrm{~g} / \mathrm{dL}$ ), osteolysis bone lesion, and renal dysfunction (eGFR $52.1 \mathrm{~mL} / \mathrm{min} / 1.73 \mathrm{~m}^{2}$ ). No cytogenetic abnormalities were detected by FISH. He was started with MP and it was administered intermittently until progression of disease. During the treatment period, he did not have any severe adverse events and maintained SD without deep response. Even after approval of novel agents, he refused to use them because of old age. He survived for 7.8 years (Table 1).

Case 3

A 73-year-old Japanese woman was diagnosed with symptomatic multiple myeloma, developed from smoldering myeloma, with IgA $\lambda$, Durie-Salmon stage IIIA, ISS II. At diagnosis, she had a PS of 0; bone marrow plasmacytosis was $6.4 \%$ and serum IgA was 1,503 mg/dL. She had mild anemia ( $\mathrm{Hb} 11.3 \mathrm{~g} / \mathrm{dL}$ ), lumbar compression fracture, and renal dysfunction (eGFR $49.8 \mathrm{~mL} / \mathrm{min} / 1.73 \mathrm{~m}^{2}$ ). The cytogenetic abnormalities were MAF:2/IGH:2 (19\%) and FGFR3:2/IGH:2 (20\%) as detected by FISH. Colon cancer was also found and she underwent operative treatment. After the operation, she refused treatment for multiple myeloma. She has been observed and has only been given bisphosphonate. She has been in SD without treatment for 8.7 years (Table 1).

\section{Case 4}

A 79-year-old Japanese woman with high blood pressure and chronic heart disease was diagnosed with symptomatic multiple myeloma IgG kappa, Durie-Salmon stage IIIA, ISS II. At diagnosis, she had PS of 0 . She had anemia ( $\mathrm{Hb} 9.4 \mathrm{mg} / \mathrm{dL}$ ), osteolysis bone lesion, and renal dysfunction (eGFR $56.9 \mathrm{~mL} / \mathrm{min} / 1.73 \mathrm{~m}^{2}$ ). She refused invasive examination and treatment because of old age. She also refused to undergo a bone marrow examination. Thus, we diagnosed her with symptomatic myeloma from the result of peripheral blood tests and the presence of bone disease. MP was started and has been continued intermittently. Even after approval of novel agents, she refused to use them. During the treatment period, she had no severe adverse events and has maintained SD without deep response. She has been in SD for 10.6 years (Table 1 ). 


\section{Case Reports in Oncology}

Nakaya et al.: Transplant-Ineligible Symptomatic but Indolent Multiple Myeloma Shows Better Prognosis with Conventional Agents

\section{Discussion}

The recent development of novel agents has markedly improved the prognosis of multiple myeloma. The treatment for symptomatic myeloma aiming for deep response is recommended because it will lead to better prognosis. Even for transplant-ineligible elderly patients, the use of novel agents is still recommended to achieve deep response. However, in clinical practice, clinicians sometimes encounter patients who show long-term prognosis without deep response. These cases have been recognized among clinicians and several reports have been published. Merchionne et al. [1] reported 21 long-term survivors who were treated with conventional chemotherapy without autologous stem cell transplant. Among these patients, the complete response (CR) rate was not correlated with overall survival (OS). Furthermore, patients with SD revealed as good a prognosis as CR or partial response (PR) patients.

Paiva et al. [2] used multiparameter flow cytometry to distinguish a MGUS (monoclonal gammopathy of undetermined significance)-like signature. The authors concluded that multiple myeloma cases with an MGUS-like signature had better prognosis and that those patients did not achieve CR and might have returned into MGUS. The authors reported that the prognosis of these patients was not dependent on the depth of response and their time to progression (TTP) was 59\% at 10 years. Sigurdardottir et al. [3] reported that myeloma progression from MGUS revealed better survival (median 2.8 years) than multiple myeloma without MGUS (2.1 years).

We speculated that our indolent myeloma patients might have been maintaining a balance between disease progression and effectiveness of chemotherapy. We hypothesized that this group might have developed symptomatic multiple myeloma slowly from MGUS or smoldering multiple myeloma (SMM) and might have immediately returned to MGUS or SMM by conventional therapy. We speculate that the underlying mechanism may be as follows. Multiple myeloma is a heterogeneity common characteristic and it is explained according to Darwinian principles: clonal evolution underlines disease progression and relapse [4]. In this respect, there is the potential for differential responses within the tumor clone and for the selection and expansion of resistant subclones. The posttreatment progression of the disease will be determined by which myeloma clones, either the more indolent clones or the more aggressive clones, repopulate the plasma cell niche in the bone marrow. In the case of the very slow progression group, we speculate that more indolent clones became dominant and more aggressive clones were eradicated by the initial therapy.

We believe that these indolent myeloma cases do not need intensive chemotherapy. Since novel agents have improved better outcome in recent clinical trials, these agents have been recommended for use [5,6]. However, this strategy might be excessive in indolent myeloma patients. For elderly patients, aggressive treatment intervention might cause infection and shorten the prognosis. Moreover, making things more complicated is the fact that the treatment is started uniformly in the presence of CRAB (hypercalcemia, renal failure, anemia, bone disease) according to the guidelines. Among CRAB, it is speculated that anemia and renal failure have poor subjective symptoms, and these factors might overlap, in contrast to myeloma. Together, this suggests that treatment intervention criteria should be carefully developed. 
Nakaya et al.: Transplant-Ineligible Symptomatic but Indolent Multiple Myeloma Shows Better Prognosis with Conventional Agents

Our cases started treatment of an initial induction therapy before approval of novel agents for use. During the treatment course, the patients received approval to begin treatment with novel agents; however, the patients' conditions were already stable and the patients did not want to switch treatments.

In conclusion, here we identified a patient group of symptomatic but indolent multiple myeloma that remained in SD with conventional therapies. These findings suggest that intensive treatment may not necessarily benefit all transplant-ineligible myeloma patients. Further studies should focus on identifying the patient group that does not need intensive treatment. One challenge is how to evaluate indolent myeloma at the time of diagnosis, as at present, there is no way to judge indolent myeloma by general examination. In addition, no trials have researched or identified prognosis factors in myeloma. However, it might be helpful to observe the clinical course or response to the treatment. This is close to the experience value of clinicians and depends on the competence of the clinicians to accurately judge the patient's condition and select treatment. Further investigations are needed to identify molecular, genetic, and biological parameters to determine indolent myeloma.

\section{Statement of Ethics}

This retrospective analysis was approved by the Ethics Committee of Kansai Medical University.

\section{Disclosure Statement}

All authors declare that there are no competing financial interests in relation to the work.

\section{References}

1 Merchionne F, Procaccio P, Dammacco F: Long-term survival in multiple myeloma: a single-center experience. Clin Exp Med 2008;8:133-139.

2 Paiva B, Vídriales MB, Rosiñol L, Martínez-López J, Mateos MV, Ocio EM, et al: A multiparameter flow cytometry immunophenotypic algorithm for the identification of newly diagnosed symptomatic myeloma with an MGUS-like signature and long-term disease control. Leukemia 2013;27:2056-2061.

-3 Sigurdardottir EE, Turesson I, Lund SH, Lindqvist EK, Mailankody S, Korde N, Björkholm M, Landgren O, Kristinsson SY, et al: The role of diagnosis and clinical follow-up of monoclonal gammopathy of undetermined significance on survival in multiple myeloma. JAMA Oncol 2015;1:168-174.

4 Morgan GJ, Kaiser MF: How to use new biology to guide therapy in multiple myeloma. Hematology Am Soc Hematol Educ Program 2012;2012:342-349. statement for the management, treatment, and supportive care of patients with myeloma not eligible for standard autologous stem-cell transplantation. J Clin Oncol 2014;32:587-600.

6 Engelhardt M, Terpos E, Kleber M, et al: European Myeloma Network recommendations on the evaluation and treatment of newly diagnosed patients with multiple myeloma. Haematologica 2014;99:232-242. 


\section{Case Reports in Oncology}

Nakaya et al.: Transplant-Ineligible Symptomatic but Indolent Multiple Myeloma Shows

Better Prognosis with Conventional Agents

Table 1. Patients' characteristics

\begin{tabular}{|c|c|c|c|c|c|c|c|c|c|c|c|c|c|c|}
\hline Case & Sex & $\begin{array}{l}\text { Age, } \\
\text { years }\end{array}$ & PH & $\begin{array}{l}\text { Survival, } \\
\text { years }\end{array}$ & Outcome & Isotype & $\mathrm{BJP}$ & PS & ISS & DS & 1 st Tx & $\begin{array}{l}\text { Re- } \\
\text { sponse }\end{array}$ & MDE & $\begin{array}{l}\text { Prior } \\
\text { status }\end{array}$ \\
\hline 1 & $\mathrm{~F}$ & 68 & CI & 6.7 & dead & IgGк & + & 0 & 2 & $3 \mathrm{~A}$ & MP & SD & anemia/bone & - \\
\hline 2 & M & 78 & HBP & 7.4 & dead & IgGK & + & 1 & 2 & $3 B$ & MP & SD & anemia/renal/bone & - \\
\hline 3 & $\mathrm{~F}$ & 73 & $\begin{array}{l}\text { Colon } \\
\text { cancer }\end{array}$ & 8.7 & alive & $\operatorname{Ig} A \lambda$ & - & 0 & 2 & $3 \mathrm{~A}$ & no Tx & SD & anemia/renal/bone & smoldering \\
\hline 4 & $\mathrm{~F}$ & 79 & $\mathrm{HBP} / \mathrm{CHF}$ & 10.6 & alive & IgGк & + & 0 & 2 & $3 \mathrm{~A}$ & MP & SD & anemia/renal/bone & - \\
\hline
\end{tabular}

PH, past history; BJP, Bence-Jones protein; PS, performance status; ISS, international staging system; DS, Durie-Salmon staging system; Tx, treatment; MDE, myeloma-defining event; F, female; M, male; CI, cerebral infarction; HBP, high blood pressure; CHF; chronic heart failure; MP, melphalan and prednisolone; SD, stable disease. 